\title{
The Effects of Cupping Massage in Patients with Chronic Neck Pain - A Randomised Controlled Trial
}

\author{
Felix J. Saha ${ }^{a}$ Stefan Schumann ${ }^{a}$ Holger Cramer ${ }^{a, b}$ Claudia Hohmann ${ }^{a}$ Kyung-Eun Choi ${ }^{a}$ \\ Roman Rolke $^{c}$ Jost Langhorst ${ }^{a}$ Thomas Rampp ${ }^{a, d}$ Gustav Dobos ${ }^{a}$ Romy Lauche ${ }^{a, b}$ \\ a Department of Internal and Integrative Medicine, Kliniken Essen-Mitte, Faculty of Medicine, University of Duisburg-Essen, Essen, Germany; \\ ${ }^{b}$ Australian Research Centre in Complementary and Integrative Medicine (ARCCIM), Faculty of Health, University of Technology Sydney, \\ Sydney, Australia; \\ ${ }^{c}$ Department of Palliative Medicine, Medical Faculty RWTH Aachen University, Aachen, Germany; \\ ${ }^{d}$ Institute for Traditional Chinese Medicine, Ayurveda and Classical Naturopathy, Kliniken Essen-Mitte, Essen, Germany
}

\section{Keywords}

Chronic neck pain · Cupping therapy .

Randomised controlled trial

\section{Summary}

Background: Chronic neck pain is a major public health burden with only limited evidence for the effectiveness of complementary therapies. This study aimed to test the efficacy of cupping massage in patients with neck pain. Patients and Methods: Patients with chronic non-specific neck pain were randomly assigned to cupping massage or a wait list control. The intervention group received 5 cupping massages on a twice-weekly basis while the control patients continued their usual treatments. The primary outcome measure was neck pain intensity $(0-100 \mathrm{~mm}$ visual analogue scale (VAS)) after 3 weeks. Secondary outcomes included pain on movement, functional disability, health-related quality of life, mechanical detection and pain thresholds and adverse events. Results: 50 patients $(52.6 \pm 10.3$ years, $92 \%$ female) were randomised to either cupping massage or a wait list ( $N=25$ each). Patients in the cupping group reported significantly less neck pain post intervention (difference per protocol $-14.3 \mathrm{~mm}$, $95 \%$ confidence interval $(\mathrm{Cl})-27.7$ to $-1.0, \mathrm{p}=0.037$; difference intention-to-treat $-10.8 \mathrm{~mm}, 95 \% \mathrm{Cl}-21.5$ to $-0.1, \mathrm{p}=0.047)$. Significant group differences in favour of the intervention were further found for pain on movement $(p=0.019)$ and functional disability $(p<0.001)$, the quality-of-life subscales pain $(p=0.002)$ and mental health $(p=0.003)$ and the mental component summary $(p=0.036)$. Changes were also found for pressure pain sensitivity at the site of maximal pain ( $p=0.022$ ). Five adverse events were reported. Conclusions: Cupping massage appears to be effective in reducing pain and increasing function and quality of life in patients with chronic non-specific neck pain. More rigorous studies are needed to confirm and extend these results.

(c) 2017 S. Karger GmbH, Freiburg

\section{Schlüsselwörter}

Nackenschmerzen · Schröpfen .

Randomisierte kontrollierte Studie

\section{Zusammenfassung}

Hintergrund: Chronische Nackenschmerzen sind eine gesundheitsökonomische Herausforderung, mit wenigen verfügbaren komplementären Therapieansätzen. In dieser Studie wurde daher die Wirksamkeit der Schröpfkopfmassage bei Patienten mit chronischen unspezifischen Nackenschmerzen untersucht. Patienten und Methoden: Die Teilnehmer wurden per Zufall entweder der Schröpfkopfmassagegruppe oder der Wartelistenkontrollgruppe zugeordnet. Die Interventionsgruppe erhielt über einen Zeitraum von 3 Wochen insgesamt 5 Behandlungen, während die Kontrollgruppe ihre üblichen Behandlungen fortführte. Der primäre Zielparameter war die Schmerzintensität nach 3 Wochen, die mittels einer $100 \mathrm{~mm}$ langen visuellen Analogskala (VAS) erfasst wurde. Sekundäre Parameter umfassten Bewegungsschmerzen, Beeinträchtigungen aufgrund der Nackenschmerzen, Lebensqualität, sensorische Wahrnehmungs- und Schmerzschwellen sowie Sicherheit. Ergebnisse: 50 Patienten $(52,6 \pm 10,3$ Jahre, 92\% Frauenanteil) wurden 2 Gruppen zugeordnet (jeweils $N=25$ ). Nach 3 Wochen berichteten die Patienten der Schröpfgruppe signifikant weniger Schmerzen in der Per-Protokoll-Analyse (Differenz $-14,3 \mathrm{~mm}, 95 \%-K o n f i d e n z i n t e r v a l l(95 \%-K I)-27,7$ bis $-1,0, \mathrm{p}=$ 0,037 ) und der Intention-to-Treat-Analyse (Differenz -10,8 mm, $95 \%-\mathrm{KI}-21,5$ bis $-0,1, p=0,047)$. Weitere Gruppenunterschiede zugunsten des Schröpfens wurden für Bewegungsschmerz $(p=$ $0,019)$ und Beeinträchtigungen $(p<0,001)$, für die Lebensqualität auf den Subskalen Schmerz $(p=0,002)$ und psychische Gesundheit $(p=0,003)$ und für den Summenwert psychische Lebensqualität $(p=0,036)$ gefunden. Veränderungen wurden weiter für die Druckschmerzschwelle am Punkt stärkster Schmerzen identifiziert $(p=0,022$ ). Fünf unerwünschte Ereignisse in der Interventionsgruppe wurden erfasst. Schlussfolgerungen: Zusammenfassend zeigte sich die Schröpfkopfmassage in der vorliegenden Studie als eine wirksame Behandlung bei chronischen Nackenschmerzen, die auch zur Verbesserung von Funktion und Lebensqualität führen kann. Weitere methodisch hochwertige Studien zum Schröpfen sind wünschenswert, um diese Befunde zu bestätigen und zu erweitern.

\section{KARGER}

(C) 2017 S. Karger GmbH, Freiburg
Dr. Romy Lauche

Department of Internal and Integrative Medicine, Kliniken Essen-Mitte

Faculty of Medicine, University of Duisburg-Essen

Am Deimelsberg 34a, 45276 Essen, Germany

r.lauche@kliniken-essen-mitte.de 


\section{Introduction}

Neck pain is a common public health problem in industrialised societies, with an average lifetime prevalence of nearly $50 \%$ (range $14.2-71.0 \%$ ) [1]. Chronic neck pain in particular not only constitutes a considerable individual burden but it is also associated with substantial work absenteeism and socioeconomic costs [2].

In a nutshell, treatment options for chronic non-specific neck pain are limited due to insufficient evidence in most cases [3]; this is also true for complementary and alternative medicine. A complementary therapeutic modality that has been the centre of several investigations within the previous years is cupping therapy. Cupping is a therapy with a long-standing history reaching back to 3300 B.C. [4], and a variety of techniques exists nowadays. The common theme in all those techniques is the utilisation of suction on the skin $[4,5]$, influencing the blood flow and microcirculation within the treated areas. Cupping therapy may be beneficial for pain conditions [6, 7], and recent trials have indicated significant effects after cupping for patients with chronic non-specific neck pain [8-12] and back pain [13-15]. The modes of action of cupping remain indeterminate, but most hypotheses for dry cupping techniques focus on the local increase of blood circulation that might lead to relieving painful muscle tension [16]. Cupping massage may combine such effects with the effects of massage when the cupping glasses are being drawn over the skin surface after the suction has been created [5].

The present study aimed to test the efficacy of 5 cupping massage treatments, compared to a wait list control group in patients with chronic non-specific neck pain.

\section{Methods}

Ethical Approval and Trial Registration

This trial was conducted between 2009 and 2011 in the Department of Complementary and Integrative Medicine in Essen, Germany. The study was approved by the ethics committee of the University Hospital Essen (approval number: 09-3987) prior to patient recruitment and was registered at ClinicalTrials.gov (registration number: NCT02651766).

\section{Design}

This trial was a randomised controlled clinical trial with 2 parallel groups. Patients were randomised to either a cupping massage group or a wait list control group and introduced to their assigned intervention. Trial measurements were repeated post intervention, 3 weeks after randomisation.

\section{Patients}

Patients were recruited via a local newspaper advertisement and website announcements. Patients interested in participating were screened by phone by a student assistant, to assess their eligibility. People who met the trial inclusion criteria were invited to receive detailed written information before their written informed consent was obtained. They then underwent physical examination by the study physician. The study physician explored the patients' medical histories and current therapies and examined their physical health and neurological function. The physician also checked the patients' medical records provided by them, e.g. laboratory findings or X-rays. If patients met the trial inclusion criteria and did not fulfil any exclusion criteria, they were included in the trial and randomised subsequently.
Trial participants were required to be between 18 and 75 years of age and to have experienced non-specific neck pain for at least the previous 3 months, for a minimum of 5 days a week. Their average neck pain intensity had to be 4 points or more on a $0-10$ numerical rating scale (NRS), with 10 being described as 'the worst pain imaginable'. The trial exclusion criteria included specific neck pain due to trauma, disc protrusion, whiplash, congenital deformity of the spine, spinal stenosis, neoplasm, inflammatory rheumatic disease or oncologic disease, and also affective disorders, addictions and psychoses requiring treatment. In addition, patients who were pregnant or who had had invasive treatment of the spine within the previous 4 weeks or spinal surgery within the previous year were excluded. Finally, patients who had initiated a new treatment regimen for neck pain within the previous 6 weeks or were planning to do so within the following 3 weeks were also excluded from the trial.

\section{Randomisation}

Patients were randomly assigned to one treatment group by using a nonstratified randomisation approach. The randomisation sequence was generated using the random number function of Microsoft ${ }^{\circledR}$ Excel software. Sequentially numbered sealed envelopes containing the patients' treatment assignments were prepared by a research coordinator who was not otherwise involved in the study. After inclusion into the study, the research fellow opened the next lowest numbered envelope to reveal the patient's treatment assignment.

\section{Interventions}

\section{Cupping Massage}

After the baseline measurement, participants in the intervention group received the first of 5 cupping massages. Cupping treatments were repeated twice weekly and performed by an experienced therapist. The patients lay prone on a massage couch with their upper torso unclothed. Their back was covered with massage oil (arnica massage oil; Weleda AG, Schwäbisch-Gmünd, Germany), and a cupping glass (diameter 3.5-5 cm; Karl Hecht GmbH, Sondheim/Rhön, Germany) was attached to the back. Negative pressure was created using the rubber ball at the top of the glass. The cup was then drawn over the skin along the spine, from the occiput towards the mid-level thoracic spine as well as over the upper trapezius muscle, maintaining the suction within the cup. The cupping massage was conducted for approximately $10 \mathrm{~min}$ and the patients were advised that the cupping massage might cause petechiae and ecchymosis being visible for days, necessitating awareness in social settings such as swimming pools.

\section{Wait List Control Group}

Treatments in the control group were not regulated; instead, patients were asked to continue their self-directed medical care. The patients were, however, asked to refrain from invasive treatments such as injections or acupuncture. They were asked not to change their treatment regimen during the course of the study. Control patients were offered the same treatment as the intervention group once the trial was concluded.

\section{Outcome Measures}

The primary outcome measure was pain intensity at rest, as recorded on a $100-\mathrm{mm}$ visual analogue scale (VAS) [17] after 3 weeks. Secondary outcome measures included functional disability, health-related quality of life and mechanical sensory and pain thresholds.

\section{Questionnaires}

To assess pain on movement, patients filled in the Pain on Movement Questionnaire (POM), where they were asked to rate the pain intensity on a $100-\mathrm{mm}$ VAS while flexing and extending their neck and laterally flexing and laterally rotating their head. An average pain-on-movement score was then calculated from all movement directions [18]. The POM has shown satisfactory validity and reliability [18]

The patients' functional neck-related disability was measured using the Neck Disability Index (NDI) [19, 20], a 10-item instrument that determines how patients feel their neck pain affecting their daily activities. Higher scores 


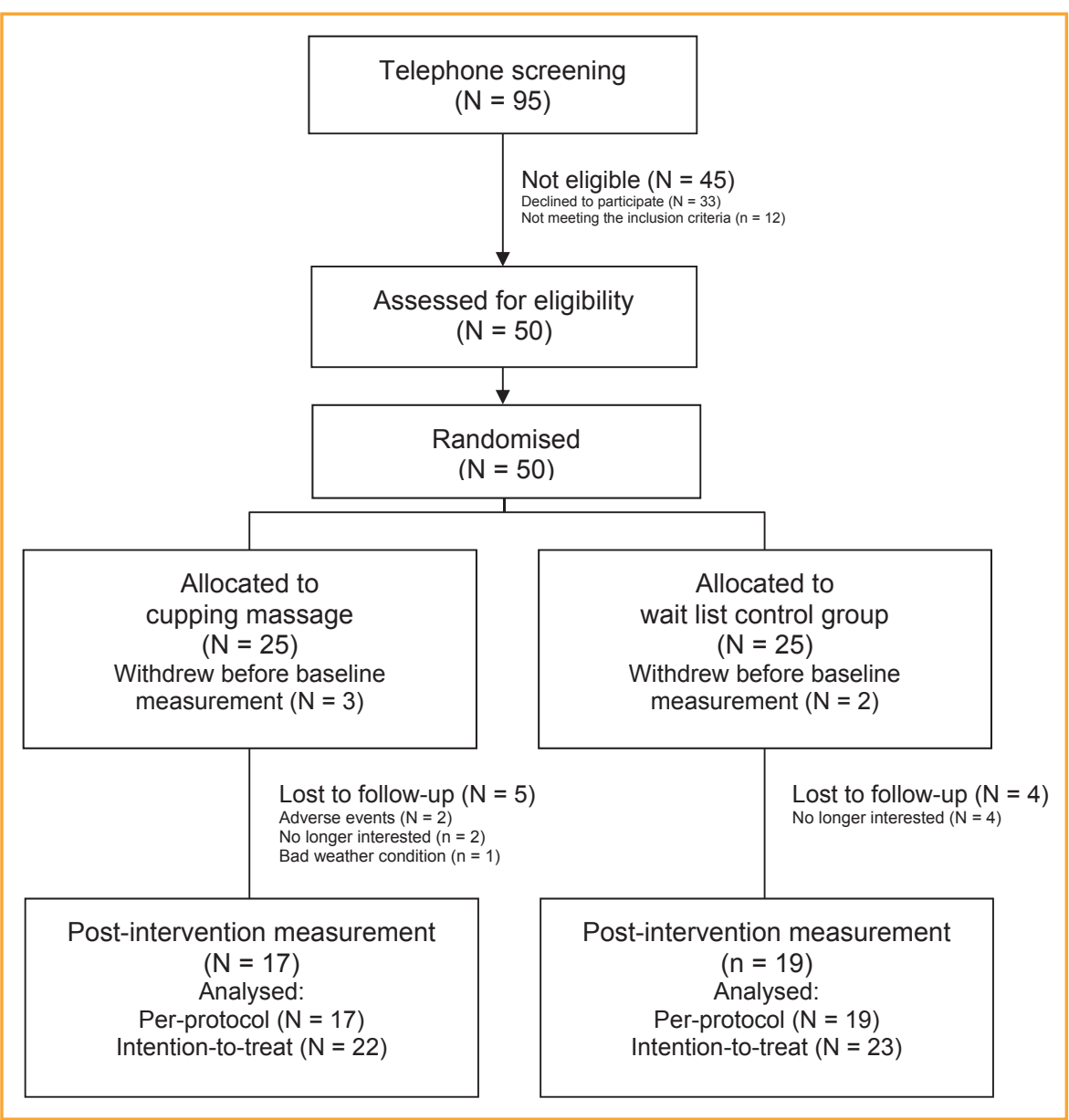

Fig. 1. Consort flow chart of patient recruitment.

The analysis of the intra-observer reliability coefficients (Pearson's r) revealed the following scores: PPT: $r=0.72$ (hand) and $r=0.76$ (foot); MDT: $\mathrm{r}=0.62$ (hand) and $\mathrm{r}=0.65$ (foot); VDT $\mathrm{r}=0.79$ (hand) and $\mathrm{r}=0.81$ (foot); and 2PD: $r=0.73$ (hand) and $r=0.69$ (foot).

\section{Patients' Expectations}

All patients rated their expectations that the cupping massage would be successful on a $100-\mathrm{mm}$ VAS, with $0 \mathrm{~mm}$ indicating 'not successful at all' and $100 \mathrm{~mm}$ indicating 'as successful as possible'.

\section{Adverse Events}

All adverse events were recorded. Patients experiencing such events were asked to see the study physician to assess their import and initiate any necessary treatment.

\section{Sample Size Calculation and Statistical Analysis}

When this study was planned, no data on the efficacy of cupping massage for chronic neck pain were available. As a consequence, a convenient sample of $\mathrm{N}=50$ participants was planned. This sample size was considered to be sufficient to detect a group difference of Cohen's $\mathrm{d}=0.8$ given a 2 -sided level $5 \%$ t-test and a statistical power of $80 \%$ even after a loss of $20 \%$ of participants during the trial.

Analyses were conducted on the 'per-protocol' as well as on the 'intention-to-treat' population for the primary outcome, including all randomised patients who had provided baseline data. Missing data were completed using the Markov chain Monte Carlo multiple imputation method in SPSS. A set of 50 imputations was generated, and the mean score was used for the analyses.

Baseline data comparability was ensured using Student's t-tests for continuous data and $\chi^{2}$ tests for categorical data. Outcome data were analysed using 
univariate analyses of covariance (ANCOVA), which modelled each post-treatment outcome as a function of the treatment group (classified factor), the patients' expectations (linear covariate) and its respective baseline value (linear covariate). All analyses were done using SPSS software (version 20.0; IBM, Copenhagen)

Table 1. Sociodemographic and baseline characteristics for the study sample

\begin{tabular}{lccc}
\hline & $\begin{array}{c}\text { Cupping } \\
(\mathrm{N}=25)\end{array}$ & $\begin{array}{c}\text { Control } \\
(\mathrm{N}=25)\end{array}$ & $\mathrm{p}$ \\
\hline Age, years & $54.3 \pm 8.6$ & $53.3 \pm 11.1$ & 0.543 \\
Gender, n (female/male) & $21 / 4$ & $25 / 0$ & 0.110 \\
Duration of neck pain, years & $7.5 \pm 6.6$ & $8.1 \pm 7.2$ & 0.772 \\
Pain intensity, mm VAS & $49.8 \pm 21.8$ & $45.1 \pm 16.3$ & 0.424 \\
Currently taking pain medication, \% & & & 0.296 \\
No medication & 43.8 & 22.2 & \\
When needed & 56.3 & 72.2 & \\
$\quad$ Regularly & 0.0 & 5.6 & \\
Treatments previously received, \% & & & 0.550 \\
$\quad$ Pain medication & 52.2 & 63.6 & 0.489 \\
Operation & 8.7 & 0.0 & 1.000 \\
Injections & 43.5 & 40.9 & 0.337 \\
Physiotherapy & 78.3 & 63.6 & 1.000 \\
Massage & 56.5 & 59.1 & 0.189 \\
Acupuncture & 39.1 & 18.2 & 0.699 \\
Chiropractic & 21.7 & 13.6 & 1.000 \\
Psychotherapy & 4.3 & 4.5 & 0.459 \\
Rehabilitation & 26.1 & 13.6 & \\
\hline
\end{tabular}

\section{Results}

\section{Patients}

The Consort flow chart of patient recruitment is shown in figure 1 . From 95 patients initially screened by telephone, 50 patients were seen by the study physician, and all of them could be enrolled. The reasons for excluding patients at screening were that they did not meet the inclusion criteria $(\mathrm{N}=33)$ or they had lost interest in the study $(\mathrm{N}=12)$.

Two patients within the intervention group and two in the control group did not provide any baseline data; they dropped out of the study before the baseline measurement. Another 6 patients in the treatment group and 4 in the control group were lost during the study for several reasons, including adverse events, loss of interest in the study or bad weather conditions. Finally, 17 patients in the intervention group and 19 in the control group were included in the per-protocol sample, and 22 and 23 , respectively, in the intention-to-treat analysis.

The patients were $52.6 \pm 10.3$ years old on average (range: 24-74 years) (table 1 ) and most of them were female (46 out of 50). They reported an average of $8.1 \pm 7.2$ years of neck pain, with the majority having tried several types of past treatment, including drug therapy (57.8\%), cervical injection (42.2\%), physical therapy (71.1\%) or massages $(57.8 \%)$, acupuncture $(28.9 \%)$, chiropractic therapy $(17.8 \%)$ or treatment in a rehabilitation centre $(20.0 \%)$. Only a minority used psychotherapy (4.4\%) or had an operation to
Table 2. Patients' pre- and post-intervention scores and estimated group differences

\begin{tabular}{|c|c|c|c|c|c|c|}
\hline & \multicolumn{2}{|l|}{ Cupping } & \multicolumn{2}{|l|}{ Control } & \multirow{2}{*}{$\begin{array}{l}\text { Estimated group } \\
\text { difference } \\
(95 \% \mathrm{CI})^{\mathrm{b}}\end{array}$} & \multirow[t]{2}{*}{$\mathrm{p}$} \\
\hline & Baseline & Week 3 & Baseline & Week 3 & & \\
\hline \multicolumn{7}{|l|}{ Pain } \\
\hline $\begin{array}{l}\text { Pain intensity (VAS) } \\
\text { (per-protocol) }\end{array}$ & $49.8 \pm 21.8$ & $29.9 \pm 22.9$ & $45.1 \pm 16.3$ & $42.8 \pm 15.8$ & $-14.3(-27.7 ;-1.0)$ & 0.037 \\
\hline $\begin{array}{l}\text { Pain intensity (VAS) } \\
\quad \text { (intention-to-treat) }\end{array}$ & & $32.3 \pm 20.0$ & & $42.4 \pm 14.7$ & $-10.8(-21.5 ;-0.1)$ & 0.047 \\
\hline Pain on movement (POM) & $41.7 \pm 22.0$ & $31.3 \pm 16.5$ & $43.1 \pm 19.2$ & $45.8 \pm 18.4$ & $-11.7(-21.3 ;-2.1)$ & 0.019 \\
\hline Functional disability (NDI) & $13.9 \pm 4.3$ & $10.3 \pm 4.2$ & $14.0 \pm 5.9$ & $13.7 \pm 5.2$ & $-4.1(-6.3 ;-2.0)$ & $<0.001$ \\
\hline \multicolumn{7}{|l|}{ Quality of life (SF-36) } \\
\hline $\begin{array}{l}\text { Physical component } \\
\text { summary }\end{array}$ & $36.4 \pm 8.1$ & $41.5 \pm 8.5$ & $40.9 \pm 7.8$ & $42.2 \pm 8.2$ & $3.1(2.3 ; 8.4)$ & 0.247 \\
\hline $\begin{array}{l}\text { Mental component } \\
\text { summary }\end{array}$ & $46.9 \pm 12.3$ & $51.2 \pm 9.2$ & $45.5 \pm 11.6$ & $45.9 \pm 10.7$ & $4.3(0.3 ; 8.4)$ & 0.036 \\
\hline Physical functioning & $65.4 \pm 17.7$ & $69.4 \pm 17.2$ & $70.8 \pm 18.7$ & $71.1 \pm 21.2$ & $4.6(-3.9 ; 13.4)$ & 0.282 \\
\hline Physical role functioning & $27.3 \pm 32.6$ & $55.0 \pm 41.4$ & $52.3 \pm 40.8$ & $57.9 \pm 37.3$ & $8.4(-20.5 ; 37.5)$ & 0.561 \\
\hline Bodily pain & $38.6 \pm 13.8$ & $54.2 \pm 19.8$ & $41.4 \pm 13.4$ & $41.9 \pm 14.0$ & $16.7(6.9 ; 26.7)$ & 0.002 \\
\hline General health perception & $58.9 \pm 16.4$ & $65.2 \pm 17.0$ & $65.4 \pm 16.5$ & $67.7 \pm 16.7$ & $6.4(-1.1 ; 13.8)$ & 0.092 \\
\hline Vitality & $44.8 \pm 19.5$ & $52.1 \pm 22.2$ & $47.0 \pm 16.9$ & $51.6 \pm 17.6$ & $5.7(-4.6 ; 15.9)$ & 0.267 \\
\hline Social role functioning & $69.6 \pm 23.8$ & $77.9 \pm 24.4$ & $68.8 \pm 24.6$ & $70.4 \pm 26.7$ & $8.5(-3.3 ; 20.3)$ & 0.154 \\
\hline Emotional role functioning & $71.0 \pm 41.8$ & $82.2 \pm 30.5$ & $58.7 \pm 42.0$ & $61.4 \pm 48.8$ & $15.6(-5.7 ; 38.8)$ & 0.138 \\
\hline Mental health & $62.7 \pm 17.3$ & $70.4 \pm 13.2$ & $66.4 \pm 16.8$ & $65.9 \pm 13.8$ & $8.5(3.1 ; 13.8)$ & 0.003 \\
\hline
\end{tabular}

${ }^{a}$ The primary outcome measure is presented as per-protocol analysis (cupping $\mathrm{N}=17$, control $\mathrm{N}=19$ ), and as per-intention-to-treat analysis (cupping $\mathrm{N}=22$, control $\mathrm{N}=23$ ); all other outcomes are based on the per-protocol population.

${ }^{b}$ Estimation results from an ANCOVA with baseline scores as covariates.

$\mathrm{CI}=$ Confidence interval; VAS = visual analogue scale; POM = Pain on Movement Questionnaire; NDI = Functional Disability Index; SF-36 = Short Form 36 Health Survey Questionnaire; ANCOVA = analysis of covariance. 
the spine (4.4\%). No differences in the patients' major sociodemographic or pain-related characteristics were found between groups at baseline (table 1).

\section{Outcome Measures}

\section{Primary Outcome}

The results of the ANCOVA are shown in table 2. A significant group difference was found for the primary outcome neck pain intensity after the intervention (difference $-14.3 \mathrm{~mm}$ VAS, 95\% confidence interval (CI) -27.7 to $-1.0 ; \mathrm{p}=0.037$ ) in the per-protocol analysis, with the group difference also being significant after the intention-to-treat analysis (difference $-10.8 \mathrm{~mm}, 95 \% \mathrm{CI}-21.5$ to $-0.1 ; \mathrm{p}=0.04758$ ).

The average pain reduction in the intervention group was $-36.2 \%$, compared to $-4.5 \%$ in the control group (difference $-31.7 \%, 95 \%$ CI -58.8 to $-4.6 ; \mathrm{p}=0.023$ ). The comparison of responders (i.e. the number of participants with a pre-defined pain reduction compared to baseline) revealed that 11 participants (64.7\%) in the cupping group, and only 1 (5.3\%) in the control group ( $\mathrm{p}<0.001)$, and 9 participants $(52.9 \%)$ in the cupping group, and again only $1(5.3 \%)$ participant in the control group ( $\mathrm{p}=$ 0.002 ), reported at least 30 and $50 \%$ pain reduction, respectively.

\section{Secondary Outcomes}

Significant group differences were further found for pain on movement (difference $-11.7 \mathrm{~mm}, 95 \% \mathrm{CI}-21.3$ to $-2.1 ; \mathrm{p}=0.019$ ), functional disability (difference $-4.1,95 \% \mathrm{CI}-6.3$ to $-2.0 ; \mathrm{p}<$ 0.001 ) and the following subscales of the SF-36 measure: bodily pain (difference 16.7 points, $95 \%$ CI 6.9 to $26.7 ; \mathrm{p}=0.002$ ), mental health (difference 8.5 points, $95 \%$ CI 3.1 to $13.8 ; \mathrm{p}=0.003$ ) and the mental component summary (difference 4.3 points, $95 \%$ CI 0.3 to 8.4; $\mathrm{p}=0.036)$.

Except for the PPT at the site of maximal pain $(\mathrm{p}=0.022)(\mathrm{On}-$ line Supplemental Table; $w w w$.karger.com/?DOI=454872), no other significant differences were found between the groups.

The patients in the intervention group were very satisfied with the treatment, and 16 out of 17 would consider using cupping massage again in the future. The average benefit reported on a $100-\mathrm{mm}$ VAS was $67.0 \pm 23.8 \mathrm{~mm}$.

\section{Safety}

In the cupping group, 5 patients reported adverse events. 2 patients experienced some headache after cupping, lasting no longer than 60 min. 1 patient complained of upper back pain, which resolved by itself after a few days without treatment. Another patient experienced some minor vertigo; it was resolved after a few days without treatment. Finally, 1 female patient in the intervention group was diagnosed with a lipoma medial to her left shoulder blade after the first cupping session, which had to be surgically removed. While the formation of a lipoma in such a short time is unlikely, cupping treatment might have triggered the visibility of the lipoma. Further information can be found in the published case report [25].

\section{Discussion}

This randomised controlled trial found that 5 cupping massage treatments reduced the pain intensity and functional disability and improved the pain-related and mental health-related quality of life in patients with chronic non-specific neck pain, compared to an untreated wait list control group. The treatment effects were also robust and significant in an intention-to-treat analysis.

Most of these results are in line with previous studies of cupping for chronic non-specific neck pain $[8,10,11]$ which found that a single traditional cupping [11], 5 applications of dry fire cupping [10] or pulsatile cupping [8] resulted in significantly better outcomes than those of the respective wait list control groups. The average pain reduction after cupping was approximately $36 \%$, which is comparable with that found in fire dry cupping (44.8\%) or pulsatile cupping (32.7\%). This represents a clinically meaningful average difference; however, the $95 \% \mathrm{CI}$ indicated that the observed effects may include clinically non-meaningful changes.

In terms of mechanical pain sensitivity, this study found an increase in the PPT only at the site of maximal pain. Since increased pressure pain sensitivity has been reported in chronic neck pain patients $[26,27]$ it might be reasonable to assume that the intervention may have influenced the pain perception at that spot. Potential modes of action of the cupping massage may include increases in local microcirculation, thus decreasing the hypersensitivity due to muscle spasm-related ischemia in the neck muscles $[12,28]$. Other hypotheses include effects on the peripheral nociceptor or at the spinal cord $[29,30]$.

Several patients reported that their neck muscles had relaxed and that the flexibility of their neck and head was increased. However, at the current point this is only speculative and not conclusive as other studies have found conflicting evidence of associations between neck pain and pressure pain sensitivity or none at all $[31,32]$.

Future trials would be needed to confirm the results of this study on the effects of cupping massage for back or neck pain. They should also investigate the specificity of effects and consider other control conditions. Since cupping massage is easy to learn and can be used in a non-clinical setting, studies investigating the efficacy in a different setting may be useful. One such study [12] was already conducted and found that 12 weeks of partner-delivered cupping massage at home was no more effective than progressive muscle relaxation. The patients were very satisfied with the possibility to conduct cupping massages at home. But this was not reflected by the results as the pain intensity did not decrease as much. Finally, more studies on the mechanics of cupping therapy are warranted [33].

This study comes with several limitations. On the one hand, several patients were lost during the study. While this may be in part related to external conditions (e.g. bad weather conditions), the withdrawal rate may also be associated with insufficient efficacy or safety issues. Overall, the withdrawal rates were comparable between the groups, ruling out such a source of bias for the most part.

A lack of a long-term follow-up, the impossibility of blinding the patients and therapists to the patients' treatment allocations 
and the lack of other control conditions such as a placebo are other potential sources of bias.

The study's strengths include the use of a randomised controlled design. The outcomes reflect the most important and recommended outcomes for chronic pain conditions, including safety. Furthermore, a standard intention-to-treat analysis was used, which confirmed the findings from the per-protocol analysis.

In conclusion, cupping massage appears to be effective in reducing pain and increasing function and quality of life in patients with chronic non-specific neck pain. More rigorous studies are needed to confirm and extend these results.

\section{Acknowledgements}

The authors want to thank Prof. Dr. Frauke Musial and Dr. Gianna Irmisch for providing support for this study during planning and conducting. They also want to express their gratitude to the Karl and Veronica Carstens Foundation for providing intellectual support.

\section{Disclosure Statement}

The authors declare that they have no conflict of interest.

\section{Author Contributions}

F.S. examined the patients and critically revised the manuscript. S.S. conducted the study and participated in data management; he supported the interpretation and drafting of the manuscript. H.C. provided support in planning and conducting the study, interpreting and drafting the manuscript. C.H. examined the patients and critically revised the manuscript. K.C. was involved in planning the study and critically revised the manuscript. R.R. analysed part of the data and critically revised the manuscript. J.L. critically revised the manuscript. T.R. was involved in planning the study; he examined patients and critically revised the manuscript. G.D. was involved in planning the study and critically revised the manuscript. R.L. planned the study and wrote the protocol, analysed and interpreted the data and drafted the manuscript.

\section{Registration}

https://clinicaltrials.gov/ct2/show/NCT02651766.

\section{Online Supplemental Material}

Online Supplemental Table Patients' pre- and post-intervention sensory and pain thresholds and estimated group differences as per protocol analysis

To access the supplemental table please refer to $w w w$.karger.com/?DOI454872.

\section{References}

1 Fejer R, Kyvik KO, Hartvigsen J: The prevalence of neck pain in the world population: a systematic critical review of the literature. Eur Spine J 2006;15:834-848.

2 Cote P, van der Velde G, Cassidy JD, Carroll LJ, HoggJohnson S, Holm LW, Carragee EJ, Haldeman S, Nordin M, Hurwitz EL, Guzman J, Peloso PM: The burden and determinants of neck pain in workers: results of the Bone and Joint Decade 2000-2010 Task Force on neck pain and its associated disorders. Spine (Phila Pa 1976) 2008;33:S60-S74.

3 Hurwitz EL, Carragee EJ, van der Velde G, Carroll LJ, Nordin M, Guzman J, Peloso PM, Holm LW, Cote P, Hogg-Johnson S, Cassidy JD, Haldeman S: Treatment of neck pain: noninvasive interventions: results of the Bone and Joint decade 2000-2010 Task Force on neck pain and its associated disorders. Spine (Phila Pa 1976) 2008;33:S123-S152.

4 Chirali I: Traditional Chinese Medicine Cupping Therapy. Philadelphia, Elsevier Churchill Livingston, 2007.

5 Abele J: Das Schröpfen: Eine bewährte alternative Heilmethode, ed 5. München, Urban und Fischer, 2003.

6 Cao H, Li X, Liu J: An updated review of the efficacy of cupping therapy. PLoS One 2012;7:e31793.

7 Cao H, Li X, Yan X, Wang NS, Bensoussan A, Liu JP: Cupping therapy for acute and chronic pain management: a systematic review of randomized clinical trials. J Tradit Chin Med Sci 2014;1:49-61.

8 Cramer H, Lauche R, Hohmann C, Choi KE, Rampp T, Musial F, Langhorst J, Dobos G: Randomized controlled trial of pulsating cupping (pneumatic pulsation therapy) for chronic neck pain. Forsch Komplementmed 2011;18:327-334.

9 Kim TH, Kang JW, Kim KH, Lee MH, Kim JE, Kim JH, Lee S, Shin MS, Jung SY, Kim AR, Park HJ, Hong KE: Cupping for treating neck pain in video display terminal (VDT) users: a randomized controlled pilot trial. J Occup Health 2012;54:416-426.
10 Lauche R, Cramer H, Choi KE, Rampp T, Saha FJ, Dobos GJ, Musial F: The influence of a series of five dry cupping treatments on pain and mechanical thresholds in patients with chronic non-specific neck pain - a randomised controlled pilot study. BMC Complement Altern Med 2011;11:63.

11 Lauche R, Cramer H, Hohmann C, Choi KE, Rampp T, Saha FJ, Musial F, Langhorst J, Dobos G: The effect of traditional cupping on pain and mechanical thresholds in patients with chronic nonspecific neck pain: a randomised controlled pilot study. Evid Based Complement Alternat Med 2012;2012:429718.

12 Lauche R, Materdey S, Cramer H, Haller H, Stange R, Dobos G, Rampp T: Effectiveness of home-based cupping massage compared to progressive muscle relaxation in patients with chronic neck pain - a randomized controlled trial. PLoS One 2013;8:e65378.

13 AlBedah A, Khalil M, Elolemy A, Hussein AA, AlQaed M, Al Mudaiheem A, Abutalib RA, Bazaid FM, Bafail AS, Essa A, Bakrain MY: The use of wet cupping for persistent nonspecific low back pain: randomized controlled clinical trial. J Altern Complement Med 2015; 21:504-508.

14 Farhadi K, Schwebel DC, Saeb M, Choubsaz M, Mohammadi R, Ahmadi A: The effectiveness of wet-cupping for nonspecific low back pain in Iran: a randomized controlled trial. Complement Ther Med 2009; 17:9-15.

15 Kim JI, Kim TH, Lee MS, Kang JW, Kim KH, Choi JY, Kang KW, Kim AR, Shin MS, Jung SY, Choi SM: Evaluation of wet-cupping therapy for persistent nonspecific low back pain: a randomised, waiting-list controlled, open-label, parallel-group pilot trial. Trials 2011;12:146.

16 Kim JI, Lee MS, Lee DH, Boddy K, Ernst E: Cupping for treating pain: a systematic review. Evid Based Complement Alternat Med 2011;2011:467014.
17 Jensen MP, Chen C, Brugger AM: Interpretation of visual analog scale ratings and change scores: a reanalysis of two clinical trials of postoperative pain. J Pain 2003;4:407-414

18 Lauche R, Cramer H, Langhorst J, Michalsen A, Dobos GJ: Reliability and validity of the Pain on Movement Questionnaire (POM) in chronic neck pain. Pain Med 2014;15:1850-1856.

19 Vernon H, Mior S: The Neck Disability Index: a study of reliability and validity. J Manipulative Physiol Ther 1991;14:409-415.

20 Cramer H, Lauche R, Langhorst J, Dobos GJ, Michalsen A: Validation of the German version of the Neck Disability Index (NDI). BMC Musculoskelet Disord 2014; 15:91.

21 Bullinger M, Kirchberger I: SF-36. Fragebogen zum Gesundheitszustand. Handanweisung. Göttingen, Hogrefe, 1998.

22 Rolke R, Baron R, Maier C, Tolle TR, Treede RD, Beyer A, Binder A, Birbaumer N, Birklein F, Botefur IC, Braune S, Flor H, Huge V, Klug R, Landwehrmeyer GB, Magerl W, Maihofner C, Rolko C, Schaub C, Scherens A, Sprenger T, Valet M, Wasserka B: Quantitative sensory testing in the German Research Network on Neuropathic Pain (DFNS): standardized protocol and reference values. Pain 2006;123:231-243.

23 Rolke R, Magerl W, Campbell KA, Schalber C, Caspari S, Birklein F, Treede RD: Quantitative sensory testing: a comprehensive protocol for clinical trials. Eur J Pain 2006;10:77-88.

24 Moseley GL: I can't find it! Distorted body image and tactile dysfunction in patients with chronic back pain. Pain 2008;140:239-243.

25 Schumann S, Lauche R, Hohmann C, Zirbes T, Dobos G, Saha FJ: [Development of lipoma following a single cupping massage - a case report]. Forsch Komplementmed 2012;19:202-205. 
26 Johnston V, Jimmieson NL, Jull G, Souvlis T: Quantitative sensory measures distinguish office workers with varying levels of neck pain and disability. Pain 2008; 137:257-265.

27 La Touche R, Fernandez-de-Las-Penas C, FernandezCarnero J, Diaz-Parreno S, Paris-Alemany A, ArendtNielsen L: Bilateral mechanical-pain sensitivity over the trigeminal region in patients with chronic mechanical neck pain. J Pain 2010;11:256-263.

28 Emerich M, Braeunig M, Clement HW, Ludtke R, Huber R: Mode of action of cupping - local metabolism and pain thresholds in neck pain patients and healthy subjects. Complement Ther Med 2014;22:148158 .
29 Musial F, Spohn D, Rolke R: Naturopathic reflex therapies for the treatment of chronic back and neck pain part 1: neurobiological foundations. Forsch Komplementmed 2013;20:219-224.

30 Spohn D, Musial F, Rolke R: Naturopathic reflex therapies for the treatment of chronic pain - part 2: quantitative sensory testing as a translational tool. Forsch Komplementmed 2013;20:225-230.

31 Lauche R, Cramer H, Langhorst J, Dobos G, Gerdle B: Neck pain intensity does not predict pressure pain hyperalgesia: re-analysis of seven randomized controlled trials. J Rehabil Med 2014;46:553-560.
32 Hubscher M, Moloney N, Leaver A, Rebbeck T, McAuley JH, Refshauge KM: Relationship between quantitative sensory testing and pain or disability in people with spinal pain - a systematic review and meta-analysis. Pain 2013;154:1497-1504.

33 Ernst E: Testing traditional cupping therapy. J Pain 2009; 10:555. 